06

\title{
Optical Solutions in Fiber Bragg Gratings with Polynomial Law Nonlinearity and Cubic-Quartic Dispersive Reflectivity*
}

(C) Elsayed M.E. Zayed ${ }^{1}$, Mohamed E.M. Alngar ${ }^{1}$, Anjan Biswas ${ }^{2,3,4,5}$, Mehmet Ekici ${ }^{6}$, Padmaja Guggilla ${ }^{5}$, Salam Khan ${ }^{5}$, Hashim Mohammad Alshehri ${ }^{3}$, and Milivoj R. Belic ${ }^{7}$

${ }^{1}$ Mathematics Department, Faculty of Science, Zagazig University, 44519 Zagazig, Egypt

${ }^{2}$ Department of Applied Mathematics, National Research Nuclear University, 115409 Moscow, Russian Federation

${ }^{3}$ Mathematical Modeling and Applied Computation (MMAC) Research Group, Department of Mathematics, King Abdulaziz University,

21589 Jeddah, Saudi Arabia

${ }^{4}$ Department of Mathematics and Applied Mathematics, Sefako Makgatho Health Sciences University, 0204 Medunsa, Pretoria, South Africa

${ }^{5}$ Department of Physics, Chemistry and Mathematics, Alabama A\&M University, Normal, AL 35762-4900, USA

${ }^{6}$ Department of Mathematics, Faculty of Science and Arts, Yozgat Bozok University, 66100 Yozgat, Turkey

${ }^{7}$ Institute of Physics Belgrade, Pregrevica 118,

11080 Zemun, Serbia

e-mail: biswas.anjan@gmail.com

Optical solitons with ber Bragg gratings and polynomial law of nonlinear refractive index are addressed in the paper. The auxiliary equation approach together with an addendum to Kudryashov's method identify soliton solutions to the model. Singular periodic solutions emerge from these integration schemes as a byproduct.

Keywords: solitons; cubic-quartic; Bragg gratings.

* Полный текст статьи опубликован в „Optics and Spectroscopy“ 2021 V. 129. N 11. 